\title{
Massa específica de polpa de açaí em função do teor de sólidos totais e da temperatura
}

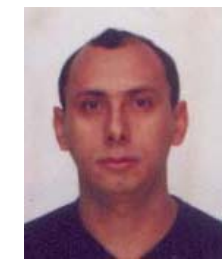

\author{
Edimir A. Pereira ${ }^{1}$, Alexandre J. de M. Queiroz ${ }^{2}$ \& Rossana M. F. de Figueirêdo ${ }^{3}$ \\ 1 Pós-Graduando, DEAg/CCT/UFCG. Fone: (83) 322-4954. E-mail: eddimir@bol.com.br (Foto) \\ 2 DEAg/CCT/UFCG. Av. Aprígio Veloso 882, CP 10.017, CEP 58109-970, Campina Grande, PB. Fone: (83) $310-1287$. \\ E-mail: alex@deag.ufpb.br \\ ${ }^{3}$ DEAg/CCT/UFCG. Fone: (83) 310-1194. E-mail: rossana@deag.ufpb.br
}

Protocolo $14-22 / 2 / 2002$

\begin{abstract}
Resumo: Desenvolveu-se este trabalho com o objetivo de se determinar a massa específica de polpa de açaí (Euterpe Oleracea, Mart.) em três concentrações (15,2, 12,5 e 9,7\%) e em cinco temperaturas $\left(10,20,30,40\right.$ e $\left.50^{\circ} \mathrm{C}\right)$. As massas específicas foram determinadas por métodos experimental e teórico. Os dados experimentais foram obtidos utilizando-se o método picnométrico. Os dados teóricos foram obtidos utilizando-se equações propostas em literatura e uma equação proposta, especificamente, para polpa de açaí. Os valores de massa específica, determinados experimentalmente, variaram entre os valores mínimo e máximo de $1.007,2$ e $1.024,9 \mathrm{~kg} \mathrm{~m}^{-3}$, respectivamente. As diferenças entre os valores experimentais e os resultados obtidos teoricamente se mantiveram, em todos os casos, abaixo de $1,2 \%$.
\end{abstract}

Palavras-chave: Euterpe oleracea Mart., propriedades termofísicas, massa específica

\section{Specific weight of "açaí" pulp as a function of total solids content and temperature}

\begin{abstract}
This work was realized to determine the specific weight of "açaí" (Euterpe Oleracea Mart.), pulp density in three concentrations (15.2, 12.5 and 9.7\%) and at five temperatures $\left(10,20,30,40\right.$ and $\left.50{ }^{\circ} \mathrm{C}\right)$. The densities were determined by experimental and theoretical methods. The experimental data were obtained using the pycnometric method. The theoretical data were obtained using models proposed in literature and by an equation proposed specifically for "açaí" pulp. The values of densities determined experimentally varied between of 1,007.2 and $1,024.9 \mathrm{~kg} \mathrm{~m}^{-3}$, respectively. The differences between the experimental values and the theoretical results in all the cases were found to be less than $1.2 \%$.
\end{abstract}

Key words: Euterpe oleracea Mart., thermophysical properties, density, açaí

\section{INTRODUÇÃO}

O açaí (Euterpe oleracea Mart.) é uma palmeira abundante no estuário do Rio Amazonas, em terrenos de várzea, igapós e terra firme, encontrando-se no Estado do Pará as maiores reservas naturais (Cavalcante, 1991; Nogueira et al., 1995).

O despolpamento comercial do fruto é obtido por meio do esmagamento e posterior filtração através de peneira fina, processo que envolve a adição de água em quantidades variadas, dependendo do produto que se quer obter (Carneiro, 2000). Na prática da extração utilizada na região produtora, a água acrescentada visa à obtenção de três concentrações distintas, com teores de sólidos totais em torno de 15,2 (Tipo A), 12,5 (Tipo B) e 9,7\% (Tipo C).

De acordo com Rogez (2000) a polpa do açaí se constitui em fonte de $\alpha$-tocoferol (vitamina E), fibras, manganês, cobre, boro e cromo. Destaca-se, dentre as frutas, quanto ao teor de lipídios, capaz de suprir cerca de $65 \%$ das necessidades teóricas recomendadas para um homem adulto. Em se tratando de proteínas, pode prover entre 25 e $65 \%$ das quantidades recomendadas; contém ainda: cálcio, magnésio, potássio e níquel; porém, é pobre (inferior a $25 \%$ do valor diário recomendado) em açúcares totais, fósforo, sódio, zinco e ferro. Conclui o autor que o açaí pode ser tido como um dos frutos mais nutritivos da Amazônia.

O processo de obtenção da polpa e o grau de beneficiamento, ainda são feitos de forma artesanal e com baixo índice tecnológico; suas características físicas, necessárias a uma exploração em nível industrial, ainda são pouco estudadas, dentre as quais se citam os dados de massa específica, essenciais no projeto de bombas, trocadores de calor, evaporadores e misturadores. 
O efeito da temperatura e da concentração na massa específica em sucos e polpas de frutas tem sido estudado por Constenla et al. (1989), Bayindirli (1993), Simões (1997), Ramos \& Ibarz (1998), Telis-Romero et al. (1998), Cepeda \& Villarán (1999) e Zainal et al. (2000).

Em vista do exposto, desenvolveu-se o presente trabalho com o objetivo de se determinar, a partir de polpa de açaí, caracterizada química e físico-quimicamente, sua massa específica em diferentes concentrações e temperaturas, comparar os valores determinados experimentalmente com os valores obtidos através da aplicação de modelos propostos em literatura e propor um modelo específico para predição desta propriedade em polpa de açaí.

\section{MATERIAL E MÉTODOS}

A etapa experimental foi conduzida no Laboratório de Armazenamento e Processamento de Produtos Agrícolas do Departamento de Engenharia Agrícola da Universidade Federal de Campina Grande. Os frutos de açaí da variedade preto, provenientes do Estado do Pará, foram colhidos no município de Abaetuba e depois transferidos para Belém, onde foram beneficiados, despolpados, congelados e transportados para a cidade de Campina Grande, PB, onde foram estocados em freezer comercial $\left(-23^{\circ} \mathrm{C}\right)$ para a coleta dos dados experimentais.

A polpa foi caracterizada por meio das seguintes determinações físico-químicas e químicas: $\mathrm{pH}$ (método potenciométrico), sólidos solúveis totais (refratômetro digital), acidez total titulável e cinzas (AOAC, 1998), lipídios, proteínas, sólidos totais, umidade, sólidos solúveis e insolúveis (Lara et al., 1976).

As massas específicas das polpas foram determinadas em cinco temperaturas, $10,20,30,40$ e $50^{\circ} \mathrm{C}$ e com teores de sólidos totais de 15,2 (Tipo A), 12,5 (Tipo B) e 9,7\% (Tipo C) e os testes realizados em triplicata, utilizando-se o picnômetro, com volume de $25 \mathrm{~mL}$ previamente calibrado com água destilada, para cada temperatura.

Para determinação das massas específicas teóricas, utilizou-se as equações de Alvarado \& Romero (1989) (Eq. 1) e de Constenla et al. (1989) (Eq. 2).

Para suco de frutas em geral, em um intervalo de temperaturas de 0 a $80^{\circ} \mathrm{C}$ e numa faixa de concentração de 5 a 30 ${ }^{\circ}$ Brix, Alvarado \& Romero (1989) desenvolveram a seguinte equação para o cálculo da massa específica:

$$
\rho=1002+4,61 B-0,460 \mathrm{~T}+7,001 \times 10^{-3} \mathrm{~T}^{2}-9,175 \times 10^{-5} \mathrm{~T}^{3}
$$

em que:

$$
\begin{array}{ll}
\rho & \text { - massa específica }\left(\mathrm{kg} \mathrm{m}^{-3}\right) \\
\mathrm{B} & \text { - concentração }\left({ }^{\circ} \mathrm{Brix}\right) \\
\mathrm{T} & \text { - temperatura }\left({ }^{\circ} \mathrm{C}\right)
\end{array}
$$

Constenla et al. (1989) propuseram uma equação para determinação da massa específica do suco clarificado de maçã, em função da concentração e da massa específica da água com coeficiente de determinação de 0,9989 e desvio padrão de 0,0021 :

$$
\rho=\rho_{\mathrm{w}} /\left(0,992417-3,7391 \times 10^{-3} \mathrm{~B}\right)
$$

em que:

$$
\begin{array}{ll}
\rho & \text { - massa específica }\left(\mathrm{kg} \mathrm{dm}^{-3}\right) \\
\rho_{\mathrm{w}} & \text { - massa específica da água }\left(\mathrm{kg} \mathrm{dm}^{-3}\right) \\
\mathrm{B} & \text { - concentração }\left({ }^{\circ} \text { Brix }\right)
\end{array}
$$

\section{Análise estatística}

Utilizou-se o delineamento inteiramente casualizado, com esquema fatorial $3 \times 5$ (três tipos e cinco temperaturas) com três repetições. Para a análise de variância foi utilizado o "software" ASSISTAT, versão 6.2 beta (Silva, 1996). A comparação entre as médias foi analisada pelo teste de Tukey em nível de $5 \%$ de probabilidade, quando o teste $\mathrm{F}$ da análise de variância se revelou significativo. As equações de regressão não linear foram obtidas utilizando-se o "software" STATISTICA, versão 5.0, para a massa específica em função da umidade e temperatura.

\section{Erros percentuais}

Para a comparação dos resultados obtidos a partir das equações encontradas em literatura com os resultados experimentais, determinaram-se os erros percentuais:

$$
\operatorname{Erro}(\%)=\left|\frac{\left(\mathrm{V}_{\text {cal }}-\mathrm{V}_{\text {exp }}\right)}{\mathrm{V}_{\text {exp }}}\right| \times 100
$$

$\begin{aligned} \text { em que: } & \\ \mathrm{V}_{\text {exp }} & \text { - valor experimental } \\ \mathrm{V}_{\text {cal }} & \text { - valor calculado }\end{aligned}$

\section{RESULTADOS E DISCUSSÃO}

Os resultados da análise físico-química e química da polpa de açaí estão apresentados na Tabela 1. Os valores experimentais estão de acordo com os padrões de identidade e qualidade para a polpa de açaí Tipo A, estabelecidos pelo Ministério da Agricultura e Abastecimento (Brasil, 2000).

Tabela 1. Caracterização físico-química e química da polpa de açaí

\begin{tabular}{lr}
\multicolumn{1}{c}{ Composição } & Valores Obtidos \\
\hline pH & $5,23 \pm 0,01$ \\
Sólidos solúveis totais ( ${ }^{\circ}$ Brix) & $4,80 \pm 0,02$ \\
Acidez total titulável (\% ácido cítrico) & $0,21 \pm 0,00$ \\
Sólidos totais (\%) & $15,27 \pm 0,04$ \\
Sólidos insolúveis (\%) & $10,41 \pm 0,10$ \\
Sólidos solúveis (\%) & $4,86 \pm 0,14$ \\
Umidade (\% base úmida) & $84,73 \pm 0,04$ \\
Cinzas (\%) & $0,64 \pm 0,01$ \\
Proteínas (\%) & $1,63 \pm 0,01$ \\
Lipídios (\%) & $6,49 \pm 0,03$ \\
\hline
\end{tabular}

De acordo com os resultados da composição físico-química e química da polpa de açaí, comprova-se o seu elevado teor nutricional, constituindo-se em excelente fonte, principalmente de lipídios e proteínas. Na Tabela 2 tem-se alguns dados da composição físico-química e química do açaí Tipo A, 
determinada experimentalmente, assim como valores encontrados na literatura. O conjunto de dados é expresso em base de sólidos totais, uma vez que a variação do teor de umidade é muito elevada, segundo os diversos pesquisadores. Observa-se, ainda, que os valores experimentais em relação aos obtidos por esses autores encontram-se na mesma faixa de $\mathrm{pH}$; o teor de sólidos totais é praticamente igual ao determinado por Rogez et al. (1996) e Carneiro (2000); no que se refere ao teor de proteínas, os valores se encontram entre os apresentados por Pontes et al. (1998) e Carneiro (2000); o conteúdo de lipídios está abaixo dos obtidos por Pontes et al. (1998) e Carneiro (2000) apresentando uma diferença apreciável quando comparado ao valor encontrado por Rogez et al. (1996) e, por fim, o teor de cinzas está próximo aos obtidos por Rogez et al. (1996) e Pontes et al. (1998) podendo-se considerar elevado quando comparado ao valor encontrado em Carneiro (2000).

Tabela 2. Composição físico-química da polpa de açaí

\begin{tabular}{lrrrr}
\hline Composição & Experimental & $\begin{array}{c}\text { Rogez } \\
\text { et al. }\end{array}$ & $\begin{array}{c}\text { Pontes } \\
\text { et al. }\end{array}$ & Carneiro \\
\hline pH & 5,20 & 5,80 & 5,00 & 5,10 \\
Sólidos totais (\%) & 15,20 & 15,00 & 12,00 & 14,97 \\
Proténas $^{1}$ & 10,75 & 13,00 & 11,54 & 9,37 \\
Lipídios $^{1}$ & 42,72 & 48,00 & 64,08 & 48,05 \\
Cinzas (\%) & 4,21 & 3,50 & 4,16 & 2,55 \\
\hline
\end{tabular}

${ }^{1} \mathrm{~g}_{100 \mathrm{~g}^{-1} \text { matéria seca }}$

$\mathrm{Na}$ Tabela 3 tem-se os resultados da análise de variância para os valores da massa específica.

Tabela 3. Resumo da análise de variância dos valores médios da massa específica de polpa de açaí

\begin{tabular}{lrrrr}
\hline \multicolumn{1}{c}{ FV } & GL & \multicolumn{1}{c}{ SQ } & QM & \multicolumn{1}{c}{ F } \\
\hline Temperatura (T) & 4 & 1386,22217 & 346,55554 & $1559,4999^{* *}$ \\
Concentração (C) & 2 & 62,13334 & 31,06667 & $139,8000^{* *}$ \\
T x C & 8 & 7,02210 & 0,87770 & $3,9500^{* *}$ \\
Resíduo & 30 & 6,66667 & 0,22222 & \\
Total & 44 & 1448,00000 & & \\
\hline
\end{tabular}

FV - Fonte de variação; GL - Grau de liberdade; SQ - Soma dos quadrados; QM - Quadrado médio; F - Variável do teste F;

- Significativo em nível de 0,01 de probabilidade

Observa-se diferença significativa entre os tratamentos, em nível de 0,01 de probabilidade pelo teste $\mathrm{F}$, para os fatores temperatura e concentração, e para a interação desses fatores, ou seja, mais de $99 \%$ de probabilidade de existir pelo menos um contraste entre médias de tratamento que difere de zero.

Os resultados experimentais obtidos da média de três repetições da massa específica da polpa de açaí nas três concentrações e cinco temperaturas estudadas estão apresentados na Tabela 4. Tem-se, também, os valores médios da interação dos fatores temperatura e concentração, os coeficientes de variação e as diferenças mínimas significativas. As amostras de açaí Tipos A, B e C, diferem estatisticamente pelo teste de Tukey em nível de 0,05 de probabilidade e, para uma mesma amostra, diferem entre as temperaturas. $O$ coeficiente de variação, em torno de $0,04 \%$, indica boa precisão experimental. Os resultados, através dos quais se observa o decréscimo da massa específica com a diminuição no teor de sólidos totais e com o aumento da temperatura em todos os casos, se assemelham aos resultados obtidos para suco de goiaba (Zainal et al., 2000), para suco de laranja (Telis-Romero et al., 1998) e polpa de manga (Simões, 1997).

Tabela 4. Valores experimentais e comparação entre médias da massa específica em diferentes concentrações e temperaturas das polpas de açaí*

\begin{tabular}{cccc}
\hline \multirow{2}{*}{$\begin{array}{c}\text { Temperatura } \\
\left({ }^{\circ} \mathrm{C}\right)\end{array}$} & \multicolumn{3}{|c}{ Massa Específica $\left(\mathrm{kg} \mathrm{m}^{-3}\right)$} \\
\cline { 2 - 4 } & Tipo A & Tipo B & Tipo C \\
\hline 10 & $1024,93 \mathrm{aA}$ & $1023,67 \mathrm{aB}$ & $1022,33 \mathrm{aC}$ \\
20 & $1023,00 \mathrm{bA}$ & $1021,70 \mathrm{bB}$ & $1020,33 \mathrm{bC}$ \\
30 & $1019,37 \mathrm{cA}$ & $1018,23 \mathrm{cB}$ & $1016,43 \mathrm{cC}$ \\
40 & $1014,43 \mathrm{dA}$ & $1013,10 \mathrm{~dB}$ & $1012,00 \mathrm{dC}$ \\
50 & $1009,80 \mathrm{eA}$ & $1008,53 \mathrm{eB}$ & $1007,17 \mathrm{eC}$ \\
\hline
\end{tabular}

Média geral $=1017,00 \mathrm{~kg} \mathrm{~m}^{-3} ;$ Coeficiente de variação $=0,046 \%$; Diferença mínima significativa colunas $=1,1186$; linhas $=0,9471$

* As médias seguidas da mesma letra minúscula nas colunas e maiúscula nas linhas não diferem estatisticamente pelo teste de Tukey, em nível de 0,05 de probabilidade

Na Figura 1 tem-se a representação gráfica simultânea da relação da massa específica com a temperatura e umidade. Observa-se a dependência da massa específica em relação à concentração dos sólidos totais e o seu decréscimo com o aumento da temperatura. Este comportamento também é mencionado por autores que trabalharam com derivados de frutas de clima temperado, como suco de maçã (Cepeda \& Villarán, 1999), suco e purê de pêssego (Ramos \& Ibarz, 1998), suco de uva Bayindirli (1993) e suco de maçã clarificado, Constenla et al. (1989). Nesses estudos, constata-se que a massa específica de suco de frutas é diretamente proporcional ao teor de umidade e inversamente proporcional à temperatura.

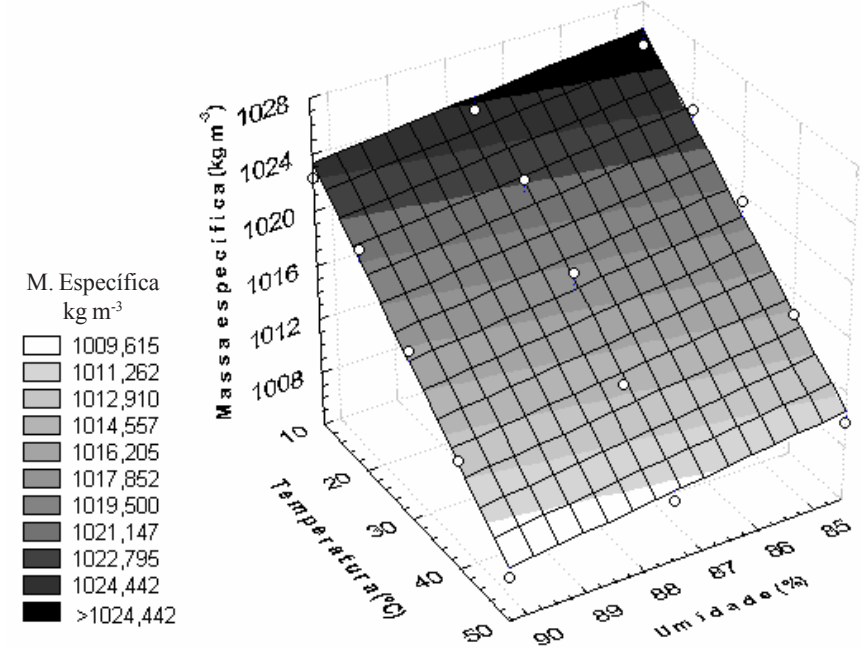

Figura 1. Massa específica experimental da polpa de açaí, em função da temperatura e do teor de umidade

Tem-se, na Tabela 5, os valores de massa específica calculados a partir de modelos propostos para os três materiais, nas cinco temperaturas estudadas, e os erros percentuais dos valores calculados em relação aos valores obtidos experimentalmente.

Observando-se os valores da Tabela 5, constata-se que todos os modelos forneceram bons ajustes em relação às 
determinações experimentais, com erros percentuais máximos em torno de $1 \%$. O ajuste obtido a partir do modelo de Constenla et al. (1989) apesar de não incluir diretamente um termo de temperatura do material, resultou nos menores erros percentuais, mostrando-se adequado para estimar os valores de massa específica de polpa de açaí em concentrações entre 3,0 e $4,8^{\circ}$ Brix com margem de erro inferior a $0,5 \%$, nas temperaturas estudadas.

Tabela 5. Valores teóricos da massa específica $\left(\mathrm{kg} \mathrm{m}^{-3}\right)$ para polpa de açaí, calculados a partir de equações propostas em literatura e erro percentual

\begin{tabular}{|c|c|c|c|c|c|c|}
\hline \multirow{2}{*}{ Polpa } & \multirow{2}{*}{ Modelos ${ }^{*}$} & \multicolumn{5}{|c|}{ Massa Específica $\left(\mathrm{kg} \mathrm{m}^{-3}\right)$} \\
\hline & & $10^{\circ} \mathrm{C}$ & $20^{\circ} \mathrm{C}$ & $30^{\circ} \mathrm{C}$ & $40^{\circ} \mathrm{C}$ & $50^{\circ} \mathrm{C}$ \\
\hline \multirow{4}{*}{$\begin{array}{l}\text { Tipo A } \\
\left(4,8^{\circ} \mathrm{B}\right)\end{array}$} & $\begin{array}{l}\text { Polpas e } \\
\text { sucos }\end{array}$ & 1020,14 & 1016,99 & 1014,15 & 1011,06 & 1007,16 \\
\hline & Erro $(\%)$ & 0,46 & 0,59 & 0,52 & 0,33 & 0,26 \\
\hline & $\begin{array}{l}\text { Suco de } \\
\text { maçã }\end{array}$ & 1025,92 & 1024,38 & 1021,77 & 1018,25 & 1013,96 \\
\hline & Erro $(\%)$ & 0,10 & 0,14 & 0,23 & 0,38 & 0,41 \\
\hline \multirow{4}{*}{$\begin{array}{c}\text { Tipo B } \\
\left(3,9^{\circ} \mathrm{B}\right)\end{array}$} & $\begin{array}{l}\text { Polpas e } \\
\text { sucos }\end{array}$ & 1015,99 & 1012,85 & 1010,00 & 1006,91 & 1003,01 \\
\hline & Erro $(\%)$ & 0,75 & 0,87 & 0,81 & 0,64 & 0,54 \\
\hline & $\begin{array}{l}\text { Suco de } \\
\text { maçã }\end{array}$ & 1022,39 & 1020,86 & 1018,25 & 1014,74 & 1010,47 \\
\hline & Erro $(\%)$ & 0,13 & 0,08 & 0,00 & 0,13 & 0,20 \\
\hline \multirow{4}{*}{$\begin{array}{l}\text { Tipo C } \\
\left(3,0^{\circ} \mathrm{B}\right)\end{array}$} & $\begin{array}{l}\text { Polpas e } \\
\text { sucos }\end{array}$ & 1011,84 & 1008,70 & 1005,85 & 1002,76 & 998,86 \\
\hline & Erro $(\%)$ & 1,02 & 1,14 & 1,04 & 0,91 & 0,83 \\
\hline & $\begin{array}{l}\text { Suco de } \\
\text { maçã }\end{array}$ & 1018,89 & 1017,36 & 1014,76 & 1011,26 & 1007,00 \\
\hline & Erro (\%) & 0,33 & 0,29 & 0,16 & 0,07 & 0,02 \\
\hline
\end{tabular}

A partir dos dados experimentais, propôs-se uma equação para o cálculo teórico da massa específica de polpa de açaí, dependente da temperatura e do teor de umidade da amostra (Eq. 4) apresentando um coeficiente de determinação $\left(\mathrm{R}^{2}\right)$ de 0,9778. Com relação aos parâmetros da equação, tem-se efeito significativo pelo teste $\mathrm{t}$ a nível de $\mathrm{p}<0,01$.

$$
\rho=1068,65-0,4579 X_{w}-0,3867 T
$$

em que:

$$
\begin{array}{ll}
\rho & \text { - massa específica }\left(\mathrm{kg} \mathrm{m}^{-3}\right) \\
\mathrm{T} & - \text { temperatura }\left({ }^{\circ} \mathrm{C}\right) \\
\mathrm{X}_{\mathrm{w}} & \text { - teor de umidade }(\%)
\end{array}
$$

Na Tabela 6 apresentam-se os valores calculados para a massa específica de polpa de açaí, de acordo com a Eq. 4, e os erros percentuais em relação aos valores experimentais.

Tabela 6. Valores teóricos da massa específica $\left(\mathrm{kg} \mathrm{m}^{-3}\right)$ para polpa de açaí, calculados pela equação proposta e erro percentual

\begin{tabular}{crrrrr}
\hline \multirow{2}{*}{ Modelo } & \multicolumn{5}{c}{ Massa Específica $\left(\mathrm{kg} \mathrm{m}^{-3}\right)$} \\
\cline { 2 - 6 } Específico & $10{ }^{\circ} \mathrm{C}$ & $20{ }^{\circ} \mathrm{C}$ & $30{ }^{\circ} \mathrm{C}$ & $40{ }^{\circ} \mathrm{C}$ & \multicolumn{1}{c}{$50{ }^{\circ} \mathrm{C}$} \\
\hline Açaí Tipo A & 1024,93 & 1023,00 & 1019,37 & 1014,43 & 1009,80 \\
Erro (\%) & 0,10 & 0,09 & 0,11 & 0,01 & 0,07 \\
\hline Açaí Tipo B & 1023,67 & 1021,70 & 1018,23 & 1013,10 & 1008,50 \\
Erro (\%) & 0,10 & 0,08 & 0,12 & 0,00 & 0,07 \\
\hline Açái Tipo C & 1022,33 & 1020,33 & 1016,43 & 1012,00 & 1007,17 \\
Erro (\%) & 0,11 & 0,07 & 0,07 & 0,02 & 0,08 \\
\hline
\end{tabular}

De acordo com a Tabela 6, verifica-se bom ajuste em relação às determinações experimentais, com erro percentual máximo de $0,11 \%$, estimando melhor os valores de massa específica de polpa de açaí, do que todas as equações sugeridas em literatura, superando, inclusive, o de Constenla et al. (1989) em treze dos quinze casos.

\section{CONCLUSÕES}

1. Os valores médios gerais experimentais da massa específica das polpas de açaí com teores de sólidos totais de $15,2,12,5$ e $9,7 \%$ e nas temperaturas de 10 a $50{ }^{\circ} \mathrm{C}$ foram, respectivamente, de $1.018,31,1.017,05$ e $1.015,65 \mathrm{~kg} \mathrm{~m}^{-3}$.

2. O modelo de Constenla et al. (1989) mostrou-se adequado para o cálculo teórico das massas específicas de açaí em todas as concentrações e temperaturas estudadas, com erro percentual máximo de $0,41 \%$ em relação aos valores determinados experimentalmente.

3. A massa específica da polpa de açaí decresceu com o aumento do teor de umidade e da temperatura, segundo uma relação quadrática com coeficiente de determinação $\left(\mathrm{R}^{2}\right)$ acima de 0,97 .

\section{LITERATURA CITADA}

Alvarado, J.D.; Romero, C.H. Physical properties of fruits: density and viscosity of juices as functions of soluble solids and content and temperature. Latin American Applied Research, Bahía Blanca, v.19, n.15, p.15-21, 1989.

AOAC - Association of Official Analytical Chemists. Official methods of analysis. Washington, 1998, 1170p.

Bayindirli, L. Density and viscosity of grape juice as a function of concentration and temperature. Journal of Food Processing \& Preservation, Trumbull, v.17, n.2, p.147-151, 1993.

Brasil. Instrução normativa n. ${ }^{\circ} 1$, de 7 de janeiro de 2000. Estabelece o regulamento técnico para a fixação dos padrões de identidade e qualidade para a polpa de fruta. Diário Oficial da República Federativa do Brasil, Brasília, 10 jan. 2000, Seção 1, n.6, p.54-58.

Carneiro, F.R.B.D. Uso da tecnologia de barreiras na obtenção da polpa de açaí e sua caracterização reológica. Campinas: UNICAMP, 2000. 135p. Tese Doutorado

Cavalcante, P.B. Frutas comestíveis da amazônia. 5.ed. Belém: CEJUP, 1991.279p. Coleção Adolpho Ducke.

Cepeda, E.; Villarán, M.C. Density and viscosity of Malus floribunda juice as a function of concentration and temperature. Journal of Food Engineering, New York, v.41, n.2, p.103-107, 1999.

Constenla, D.T.; Lozano, J.E.; Crapiste, G.H. Thermophysical properties of clarified apple juice as a function of concentration and temperature. Journal of Food Science, Chicago, v.54, n.3, p.663-668, 1989. 
Lara, A.B.W.H.; Nazário, G.; Almeida, M.E.W.; Pregnolato, W. Normas analíticas do Instituto Adolfo Lutz: métodos químicos e físicos para análises de alimentos. 2. ed. São Paulo: Melhoramentos, 1976.371p.

Nogueira, O.L.; Carvalho, C.J.R.; Muller, C.H.; Galvão, E.U.P.; Silva, H.M.E.; Rodrigues, J.E.L.F.; Oliveira, M.S.P.; Carvalho, J.E.U.; Rocha Neto, O.G.; Nascimento, W.M.O.; Calzavara, B.B.G. A cultura do açaí. Brasília: EMBRAPA, 1995. 49p.

Pontes, M.A.N.; Magno, P.S.L.; Felipe, A.M.P.; Mattieto, R.A. Elaboração do néctar de açaí (Euterpe oleracea Mart.) com e sem ácido cítrico, In: Congresso Brasileiro de Ciência e Tecnologia de Alimentos, 16, 1998. Rio de Janeiro. Anais... Rio de Janeiro: SBCTA, 1998. CD Rom.

Ramos, A.M.; Ibarz, A. Density of juice and fruit puree as a function of soluble solids content and temperature. Journal of Food Engineering, New York, v.35, n.1, p.57-63, 1998.

Rogez, H. Açaí: preparo, composição e melhoramento da conservação. Belém: EDUFPA, 2000.313p.
Rogez, H.; Buxant, R.; Mignolet, E.; Givron, C.; Pascal, S.; Ribeiro, C.; Larondelle, Y. Chemical composition of the edible parts of four typical Amazonian fruits: Assai, Araca, Bacuri, Cupuassu. Journée Universitaire de Recherche en Nutrition, Louvain-la-Neuve, v.3, p.10-15, 1996.

Silva, F. de A.S. The ASSISTAT software: statistical assistence. In: International Conference on Computers in Agriculture, 6, 1996, Cancun. Anais.... Cancun: American Society of Agricultural Engineers, 1996. p.294-298.

Simões, R. M. Propriedades termofísicas da polpa de manga. Campinas: UNICAMP, 1997. 93p. Dissertação Mestrado

Telis-Romero, J.; Telis, V.R.N.; Gabas, A.L.; Yamashita, F. Thermophysical properties of Brazilian orange juice as affected by temperature and water content. Journal of Food Engineering, New York, v.38, n.1, p.27-40, 1998.

Zainal, B.S.; Abdul Rahman, R.; Ariff, A.B.; Saari, B.N.; Asbi, B.A. Effects of temperature on the physical properties of pink guava juice at two different concentrations. Journal of Food Engineering, New York, v.43, n.1, p.55-59, 2000. 